\title{
Optimal Energy Transfer in Band-Limited Communication Channels
}

\author{
Michele Elia, Senior Member, IEEE, Giorgio Taricco, Member, IEEE, and Emanuele Viterbo, Member, IEEE
}

\begin{abstract}
Maximization of the energy transfer ratio for timelimited signals over linear channels is considered. The case of linear channels with rational transfer function is addressed and a general procedure for the analytic solution of the maximization problem is outlined. The maximum energy transfer ratio over a fixed time interval is evaluated in some cases of interest. Two performance metrics-the energy transfer ratio and the energy intersymbol interference (ISI) ratio-are evaluated for optimal signals and compared to those of commonly used rectangular and sinusoidal pulses in order to determine the achievable gain.
\end{abstract}

Index Terms - Band-limited channels, energy transfer optimization, multidimensional signals.

\section{INTRODUCTION}

$\mathbf{F}$ OURIER analysis shows that time-limited signals sent through band-limited channels are spread in time. The pulse energy dispersion is a source of performance degradation which is partially recovered in many applications by equalization techniques. These countermeasures would certainly be more efficient if used jointly with signals which have a minimal energy spread outside the signaling interval by filtering. This optimization problem attracted the attention of many researchers early in the 1950's because of its implications for digital communications, system theory, and related fields. Much of this work was devoted to the ideal low-pass filter on account of its natural connection with the representation of band-limited signals [14], [6], [7]. A further motivation was to provide "optimal" finite sets of orthogonal signals of limited time duration $T$ to be used in the design of digital signals for sending information in the then emerging global digital communication system [13]. In these applications, the energy spread outside the interval $I=[0, T]$ produces disturbances known as intersymbol interference (ISI). Hence, the maximum signal energy transferred at the filter output was considered an important issue in the design of good signal sets for digital transmissions. This energy transfer was measured as the output-to-input signal energy ratio over potentially different time intervals (in this paper we denote by $I$ and $J$ the input and output time interval, respectively). All classical works refer to the unlimited output time interval $J=(-\infty, \infty)$ and, with this assumption, Slepian [14] solved the problem for the ideal

Manuscript received October 1, 1996; revised November 1, 1998. The material in this paper was presented in part at Seizième Colloque sur le Traitement du Signal et des Images (GERTSI'97), Grenoble, France, September 1997.

The authors are with the Dipartimento di Elettronica, Politecnico di Torino, I-10129 Torino, Italy.

Communicated by N. Seshadri, Associate Editor for Coding Techniques.

Publisher Item Identifier S 0018-9448(99)06033-2. low-pass channel. Specifically, he showed that the optimal pulses are related to prolate spheroidal wave functions, and that these signals are also optimal over the limited output time interval $J=I$. This property does not hold for all channels, as we will show in this paper. Subclasses of rational transfer function channels were considered as well as the ideal lowpass channel. For instance, in [4] the optimal waveforms were found for the single-pole low-pass filter and in [2] and [3] the optimal waveforms were found for Butterworth filters referring to the output time interval $(-\infty, \infty)$. In these papers, special analytic techniques were developed which can be derived from Youla's general approach [17].

Undoubtedly, the case of limited output time interval $J$ is the most interesting because it gives the minimum achievable ISI for a fixed channel bandwidth $W$ and symbol time duration $T$. It has, however, received little attention probably due to the fact that analytical solutions seemed unlikely. In this paper, the maximization problem will be addressed for an output interval $J=I=[0, T]$ and for its delayed version $J=\left[t_{0}, t_{0}+T\right]=$ $t_{0}+I$. The introduction of a delay $t_{0}$ may lead to significant reduction of the ISI without increasing the complexity of the system. Delays often occur in digital communications and are managed routinely. The computation of the minimum time offset $t_{0}$ yielding the maximum transfer of energy will be carried out as part of the optimization process.

This paper is organized as follows. In Section II we develop basic theoretical aspects of the optimization problem concerning both finite and infinite output intervals $J$. We define the energy transfer ratio and the energy ISI ratio, two figures of merit which will be used to compare optimal and standard signal sets. In Section III we consider channels with rational transfer function which are physically realizable and we find the general solution for finite output time intervals. In Section IV we collect numerical results obtained from closed-form solutions for the ideal low-pass channel and channels with rational transfer function of order one and two. Also, we compute the energy transfer ratio and the energy ISI ratio for the optimal, the "rectangular," and the "sinusoidal" signal sets. Finally, Section V is devoted to conclusions and comments as well as remarks on possible applications.

\section{General Results}

In this study we consider square-integrable functions $y(t) \in$ $L^{2}(R)$ over the real axis $R=(-\infty, \infty)$. The energy $\mathcal{E}_{J}[y]$ of $y(t)$ included in the interval $J$ is given by the integral

$$
\mathcal{E}_{J}[y]=\int_{J} y(t)^{2} d t
$$


Whenever $y(t)$ is the output of a linear channel with impulse response $h(t, \tau)$ and input signal $x(t) \in L^{2}(I)$, we have

$$
y(t)=\int_{I} h(t, \tau) x(\tau) d \tau, \quad t \in R .
$$

The energy of $y(t)$ included in $J \subset R$ can be expressed as a quadratic integral form in $x(t)$

$$
\mathcal{E}_{J}[y]=\int_{J} y(t)^{2} d t=\int_{I} \int_{I} \mathcal{K}_{J}(t, \tau) x(t) x(\tau) d t d \tau
$$

where the energy kernel is defined as the integral

$$
\mathcal{K}_{J}(t, \tau)=\int_{J} h(\theta, t) h(\theta, \tau) d \theta .
$$

Quadratic forms are always present when we consider the energy of signals connected by linear transformations such as filtering. The filtered signals are spread in time, and the resulting energy dispersion is usually an unwanted side-effect. Two suitable measures for this effect are the energy transfer ratio and the energy ISI ratio.

Definition 1: The energy transfer ratio from $I$ to $J$ for a channel with impulse response $h(t, \tau)$, input $x(t), t \in I$, and output $y(t), t \in J$, is defined as

$$
\lambda[x, J, h]=\frac{\mathcal{E}_{J}[y]}{\mathcal{E}_{I}[x]} .
$$

The energy ISI ratio is defined as

$$
\eta[x, J, h]=\frac{\mathcal{E}_{R}[y]-\mathcal{E}_{J}[y]}{\mathcal{E}_{J}[y]} .
$$

When $J=I$, the energy transfer ratio is defined in such a way that the greater the ratio, the greater the energy passing the filter. Hence, for channels with additive noise, optimization of this figure increases the signal-to-noise ratio. The energy ISI ratio, on the other hand, is a measure of the amount of ISI because it directly compares the energy included in the signaling time interval with the energy spread outside it.

The impulse response $h(t, \tau)$ is normalized to obtain the condition

$$
\max _{x \in L^{2}(R)} \lambda[x, J, h] \leq 1 .
$$

If the channel is time-invariant with impulse response $h(t-\tau)$ and Fourier transform $H(f)=\mathcal{F}\{h(\theta)\}$, then Parseval's identity implies that constraint (4) is equivalent to $\max _{f}|H(f)|^{2} \leq 1$. In the light of the above assumptions and definitions, the optimization problem we are concerned with can be stated as follows.

Problem 1: Given a linear channel with impulse response $h(t, \tau)$, find a signal $x(t) \in L^{2}(I)$ with fixed energy $\mathcal{E}_{I}[x]=\mathcal{E}$ which gives the maximum $\lambda_{\text {opt }}$ of $\lambda[x, J, h]$.

Necessary, and in many cases sufficient, conditions characterizing any solution $x(t)$ are already known [4], [8]. In fact, a solution to Problem 1 is a signal $x(t)$ satisfying the following Fredholm integral equation of the second kind

$$
\int_{I} \mathcal{K}_{J}(t, u) x(u) d u=\lambda x(t), \quad t \in I
$$

with a positive-definite kernel, as defined in (3). The greatest eigenvalue $\lambda[x, J, h]$ gives the maximum energy transfer ratio.

When $J=t_{0}+I$, the optimization procedure involves two steps. First, $t_{0}$ is fixed and the optimum is computed by solving the Fredholm integral equation (5), then the best $t_{0}$ is found using the following proposition.

Proposition 2.1: The solution of Problem 1 when $J=$ $t_{0}+I$ is given by a normalized eigenfunction $x(t)$ of $\mathcal{K}_{J}(t, u)$ defined in (3)

$$
\int_{I} \mathcal{K}_{J}(t, \tau) x(\tau) d \tau=\lambda x(t), \quad t \in I
$$

and $t_{0}$ is the minimum nonnegative value obtained from the condition

$$
y\left(t_{0}\right)^{2}=y\left(t_{0}+T\right)^{2} .
$$

Proof: In this case the kernel is

$$
\mathcal{K}_{J}(u, v)=\int_{t_{0}}^{t_{0}+T} h(t, u) h(t, v) d t .
$$

Equating to zero the derivative of the output energy $\mathcal{E}_{J}[y]$ with respect to $t_{0}$

$$
\frac{\partial}{\partial t_{0}}\left\{\int_{I} \int_{I} x(u) x(v) \int_{t_{0}}^{t_{0}+T} h(t, u) h(t, v) d t d u d v\right\}=0
$$

where the contributions due to the derivatives of $x(u)$ and $x(v)$ with respect to $t_{0}$ can be neglected because

$$
\begin{aligned}
& \int_{I} \int_{I} x(u) \frac{\partial x(v)}{\partial t_{0}} \int_{t_{0}}^{t_{0}+T} h(t, u) h(t, v) d t d u d v \\
& \quad=\int_{I} \int_{I} x(u) \frac{\partial x(v)}{\partial t_{0}} \mathcal{K}_{J}(u, v) d u d v \\
& \quad=\lambda \int_{I} x(v) \frac{\partial x(v)}{\partial t_{0}} d v=\frac{\lambda}{2} \frac{\partial}{\partial t_{0}} \int_{I}(x(v))^{2} d v=0 .
\end{aligned}
$$

Thus we get the following necessary condition for a maximum:

$$
y\left(t_{0}+T\right)^{2}-y\left(t_{0}\right)^{2}=0 .
$$

This equation may have more than one solution, which may be either positive or negative. As we are interested in the smallest delay, we keep the smallest nonnegative value for $t_{0}$.

Remarks: In view of Proposition 2.1, $t_{0}$ is a characteristic figure of every time-invariant linear channel. It can be conveniently assumed as a measure of the delay of a digital signal instead of the commonly used group delay. Moreover, in contrast to the group delay, $t_{0}$ does not depend on frequency.

\section{A. Time-Invariant Linear Channels}

The impulse response of a time-invariant linear channel is $h(\tau)$, thus the input-output relationship (1) is a convolution, and the kernel (3) is characterized using a Fourier transform when $J=R$

$$
\mathcal{K}_{J}(u, v)=k(u-v) \quad \text { and } \quad k(\tau)=\mathcal{F}^{-1}\left\{|H(f)|^{2}\right\} .
$$


Proposition 2.2: With the assumptions of Proposition 2.1, if $h(t)$ is an even function of $t$, then $t_{0}=0$ is the minimum delay that gives the maximum energy transfer. Additionally, we have $x(t)=x(T-t)$ and $y(t)=y(T-t)$.

Proof: We show that if $t_{0}=0$, the condition (7) is satisfied. First, if $t_{0}=0$ and $h(t)=h(-t)$, we have

$$
\begin{aligned}
\mathcal{K}_{J}(u, v) & =\int_{0}^{T} h(t-u) h(t-v) d t \\
& =\int_{0}^{T} h(u-t) h(v-t) d t \\
& =\int_{0}^{T} h(u-T+t) h(v-T+t) d t \\
& =\mathcal{K}_{J}(T-u, T-v) .
\end{aligned}
$$

Then, if $x(t)$ is a solution of (6), so is $x(T-t)$

$$
\begin{aligned}
\int_{0}^{T} & \mathcal{K}_{J}(t, u) x(T-u) d u \\
& =\int_{0}^{T} \mathcal{K}_{J}(T-t, T-u) x(T-u) d u \\
& =\int_{0}^{T} \mathcal{K}_{J}(T-t, u) x(u) d u=\lambda x(T-t) .
\end{aligned}
$$

Therefore, $x(T-t)$ can only differ from $x(t)$ by a constant factor which must be 1 on setting $t=T / 2$. Hence, $x(t)=$ $x(T-t)$ and we have

$$
\begin{aligned}
y(T-t) & =\int_{0}^{T} x(\tau) h(T-t-\tau) d \tau \\
& =\int_{0}^{T} x(T-\tau) h(T-t-\tau) d \tau \\
& =\int_{0}^{T} x(u) h(u-t) d u=y(t)
\end{aligned}
$$

which holds for any $t$. In particular, equality $y(T)=y(0)$ together with condition (7) yields $t_{0}=0$ as minimum delay.

This theorem shows that for time-invariant linear channels with time-symmetric (even) impulse response the maximum energy transfer over a finite output interval $J=t_{0}+I$ occurs for $t_{0}=0$. Although these transfer functions are not physically realizable, they include important filters such as the ideal lowpass filter. Therefore, Slepian's optimal solution for $J=I$ is also optimal for $J=t_{0}+I$ with $t_{0}=0$.

\section{Channels with Rational Transfer Functions}

In this section we consider channels with a rational transfer function and solve Problem 1 for a finite output time interval $J=\left[0, T_{o}\right]$. Let the filter rational transfer function be given as a Laplace transform

$$
H(s)=\mathcal{L}\{h(\tau)\}=\frac{1+h_{1} s+\cdots+h_{m} s^{m}}{1+g_{1} s+\cdots+g_{n} s^{n}}=\frac{M(s)}{P(s)}
$$

with $n>m$, and let us assume that $H(s)$ has simple poles $\alpha_{1}, \cdots, \alpha_{n}$. The impulse response is consequently

$$
h(t)=u(t) \sum_{i=1}^{n} A_{i} e^{\alpha_{i} t}
$$

where $u(t)$ denotes the unit step function.
Our aim is to find the sequences of eigenfunctions $x_{n}(t)$ and eigenvalues $\lambda_{n}$ which satisfy the Fredholm integral equation of Problem 1. The case of an infinite output time interval has been settled by several authors [13], [14], [2], [4] who developed various techniques based on the common principle that commuting operators admit the same set of eigenfunctions [1]. In particular, if $H(s)$ has simple poles, Youla's solution [17] (see Appendix B) exploits the rational property of the transfer function and reduces the labor for solving a transcendental equation which cannot be further simplified. Although Youla's simplification does not apply to finite $J$, most of the arguments still work if combined with Franks' approach [3]. The method results in a three step program.

Step 1: Find a differential operator which commutes with the Fredholm integral operator.

Step 2: Obtain a candidate eigenfunction solving a differential equation.

Step 3: Obtain a transcendental equation for the eigenvalues substituting the candidate eigenfunction into (5).

Now let us describe each step of this program in detail.

Step 1: Given that $J=\left[0, T_{\circ}\right]$, the kernel $\mathcal{K}_{J}(t, \tau)$ can be written as a difference of the kernel $\mathcal{K}_{\infty}(|t-\tau|)$ for infinite $J$ and a residue $\mathcal{K}_{\text {res }}(t, \tau)$, that is,

$$
\mathcal{K}_{J}(t, \tau)=\mathcal{K}_{\infty}(|t-\tau|)-\mathcal{K}_{\text {res }}(t, \tau)
$$

where

$$
\begin{aligned}
\mathcal{K}_{\infty}(|t-\tau|) & =\int_{0}^{\infty} h(\theta-t) h(\theta-\tau) d \theta, \\
\mathcal{K}_{\text {res }}(t, \tau) & =\int_{T_{o}}^{\infty} h(\theta-t) h(\theta-\tau) d \theta,
\end{aligned}
$$

The time-invariant kernel $\mathcal{K}_{\infty}(|t-\tau|)$ has Laplace transform

$$
K(s)=H(s) H(-s)=\frac{N\left(s^{2}\right)}{D\left(s^{2}\right)}
$$

and can be expressed by means of exponentials depending only on the roots of $P(s)$.

$$
\begin{aligned}
\mathcal{K}_{\infty}(|t-\tau|)= & \int_{0}^{\infty} h(\theta-t) h(\theta-\tau) d \theta \\
= & \int_{\max \{t, \tau\}}^{\infty} \sum_{i, j} A_{i} A_{j} e^{\left(\alpha_{i}+\alpha_{j}\right) \theta-\alpha_{i} t-\alpha_{j} \tau} d \theta \\
= & \sum_{i, j}-\frac{A_{i} A_{j}}{\alpha_{i}+\alpha_{j}} e^{\left(\alpha_{i}+\alpha_{j}\right)(\max \{t, \tau\})-\alpha_{i} t-\alpha_{j} \tau} \\
= & \sum_{i, j}-\frac{A_{i} A_{j}}{\alpha_{i}+\alpha_{j}} e^{\frac{\alpha_{i}+\alpha_{j}}{2}|t-\tau|-\frac{\alpha_{i}-\alpha_{j}}{2}(t-\tau)} \\
= & -\sum_{i} \frac{A_{i}^{2}}{2 \alpha_{i}} e^{\alpha_{i}|t-\tau|}-2 \sum_{i<j} \frac{A_{i} A_{j}}{\alpha_{i}+\alpha_{j}} e^{\frac{\alpha_{i}+\alpha_{j}}{2}|t-\tau|} \\
& \times \cosh \left(\frac{\alpha_{i}-\alpha_{j}}{2}|t-\tau|\right)
\end{aligned}
$$

where the clearly time-invariant expression has been obtained by using the identity

$$
2 \max \{t, \tau\}=|t-\tau|+t+\tau .
$$


The residue $\mathcal{K}_{\text {res }}(t, \tau)$ can also be expressed as a sum of exponentials

$$
\mathcal{K}_{\text {res }}(t, \tau)=\sum_{i, j} \frac{A_{i} A_{j}}{\alpha_{i}+\alpha_{j}} e^{\left(\alpha_{i}+\alpha_{j}\right) T_{o}-\alpha_{i} t-\alpha_{j} \tau}
$$

and it is clearly not time-invariant.

Let $D\left(d^{2} / d t^{2}\right)$ denote a linear differential operator associated to the denominator of $K(s)$, then the following properties of $\mathcal{K}_{\infty}(|t-\tau|)$ and $\mathcal{K}_{\text {res }}(t, \tau)$ can be derived.

1) $\mathcal{K}_{\infty}(|t-\tau|)$ possesses continuous derivatives of all order for $t \neq \tau$.

2)

$$
D\left(\frac{d^{2}}{d t^{2}}\right) \mathcal{K}_{\infty}(|t-\tau|)=0, \quad t \neq \tau
$$

3)

$$
D\left(\frac{d^{2}}{d t^{2}}\right) \mathcal{K}_{\mathrm{res}}(t, \tau)=0
$$

4) From the van der Pol and Bremmer breakup [9]

$$
\begin{aligned}
\lambda x(t)= & \int_{0}^{t} \mathcal{K}_{\infty}(t-\tau) x(\tau) d \tau+\int_{t}^{T} \mathcal{K}_{\infty}(\tau-t) x(\tau) d \tau \\
& -\int_{0}^{T} \mathcal{K}_{\text {res }}(t, \tau) x(\tau) d \tau, \quad t \in I
\end{aligned}
$$

of (5) and the continuity properties of $\mathcal{K}_{\infty}(\theta)$ it follows that $x(t)$ possesses continuous derivatives of all orders in the open interval $I$.

5) Following [4], let us consider the differential operator $\left[1-D\left(\frac{d^{2}}{d t^{2}}\right)\right]$ which, applied to the integral equation

$$
\lambda \psi(t)=\int_{0}^{T} \mathcal{K}_{\infty}(|t-\tau|) \psi(\tau) d \tau
$$

produces the differential equation

$$
\begin{aligned}
{\left[1-D\left(\frac{d^{2}}{d t^{2}}\right)\right] \lambda \psi(t)=} & \int_{0}^{T} \mathcal{K}_{\infty}(|t-\tau|) \psi(\tau) d \tau \\
& -N\left(\frac{d^{2}}{d t^{2}}\right) \psi(t)
\end{aligned}
$$

6) Since the operator $\left[1-D\left(\frac{d^{2}}{d t^{2}}\right)\right]$ leaves the integral

$$
\int_{0}^{T} \mathcal{K}_{\text {res }}(t, \tau) \psi(\tau) d \tau
$$

unchanged, it follows that the same differential operator applied to the integral equation for $J=\left[0, T_{o}\right]$ yields the differential equation

$$
\left[1-D\left(\frac{d^{2}}{d t^{2}}\right)\right] \lambda x(t)=\lambda x(t)-N\left(\frac{d^{2}}{d t^{2}}\right) x(t)
$$

that is,

$$
\left(D\left(\frac{d^{2}}{d t^{2}}\right)-\frac{1}{\lambda} N\left(\frac{d^{2}}{d t^{2}}\right)\right) x(t)=0 .
$$

Step 2: Let $\pm \sigma_{1}, \cdots, \pm \sigma_{n}$ be the $2 n$ roots of the characteristic equation

$$
D\left(s^{2}\right)-\frac{1}{\lambda} N\left(s^{2}\right)=0 .
$$

Then the eigenfunctions for $\mathcal{K}_{J}(t, \tau)$ are of the form

$$
x(t)=\sum_{i=1}^{n}\left[C_{\ell} e^{\sigma_{i} t}+D_{\ell} e^{-\sigma_{i} t}\right], \quad t \in I .
$$

Step 3: Substitute the candidate eigenfunction of the form (11) into (5) and obtain the following homogeneous linear system of $2 n$ equations in the $2 n$ unknowns $C_{\ell}, D_{\ell}, \ell=$ $1, \cdots, n$ :

$$
\left\{\begin{array}{l}
\sum_{j, \ell} C_{\ell} \frac{A_{j}}{\left(\alpha_{j}+\alpha_{i}\right)\left(\alpha_{i}-\sigma_{\ell}\right)} \\
\quad+\sum_{j, \ell} D_{\ell} \frac{A_{j}}{\left(\alpha_{j}+\alpha_{i}\right)\left(\alpha_{i}+\sigma_{\ell}\right)}=0 \\
\sum_{j, \ell} C_{\ell}\left(\frac{A_{j}}{\left(\alpha_{i}+\alpha_{j}\right)\left(\alpha_{j}-\sigma_{\ell}\right)}-\frac{A_{j} e^{\left(\alpha_{i}+\sigma_{\ell}\right) T}}{\left(\alpha_{i}+\alpha_{j}\right)\left(\alpha_{i}+\sigma_{\ell}\right)}\right. \\
\left.-e^{\left(\alpha_{i}+\alpha_{j}\right) T_{o}} \frac{A_{j}\left(e^{\left(-\alpha_{j}+\sigma_{\ell}\right) T}-1\right)}{\left(\alpha_{i}+\alpha_{j}\right)\left(\alpha_{j}-\sigma_{\ell}\right)}\right) \\
+\sum_{j, \ell} D_{\ell}\left(\frac{A_{j}}{\left(\alpha_{i}+\alpha_{j}\right)\left(\alpha_{j}+\sigma_{\ell}\right)}-\frac{A_{j} e^{\left(\alpha_{i}-\sigma_{\ell}\right) T}}{\left(\alpha_{i}+\alpha_{j}\right)\left(\alpha_{i}-\sigma_{\ell}\right)}\right. \\
\left.-e^{\left(\alpha_{i}+\alpha_{j}\right) T_{o}} \frac{A_{j}\left(e^{\left(-\alpha_{j}-\sigma_{\ell}\right) T}-1\right)}{\left(\alpha_{i}+\alpha_{j}\right)\left(\alpha_{j}+\sigma_{\ell}\right)}\right)=0
\end{array}\right.
$$

for $i=1, \cdots, n$. Looking for nonzero solutions, the determinant of the coefficient matrix must be zero, a condition which yields the transcendental equations for the eigenvalues. Finally, the nonzero solution of the system gives the coefficients characterizing the eigenfunctions up to a scale factor.

As an illustrative example, we apply the above procedure to the single-pole linear channel.

Example 1-Single-Pole Linear Channel and Finite J: The transfer function of this channel is

$$
H(s)=\frac{1}{1+s /(2 \pi W)}
$$

where $W$ is the $3-\mathrm{dB}$ bandwidth. The impulse response for this channel is

$$
h(t)=2 \pi W u(t) e^{-2 \pi W t}
$$

where $u(t)$ denotes the unit step function. The Laplace transform of the energy kernel for $J=R$ is the ratio of the two polynomials:

$$
N\left(s^{2}\right)=1 \quad \text { and } \quad D\left(s^{2}\right)=1-\frac{s^{2}}{(2 \pi W)^{2}} .
$$

Let us assume that input signals have duration $T$ and the finite output time interval is $J=\left[0, T_{o}\right]$. Hence the energy kernel

$$
\begin{aligned}
\mathcal{K}(t, \tau)= & \int_{0}^{T_{o}} 2 \pi W u(\theta-t) e^{-2 \pi W(\theta-t)} \\
& \times 2 \pi W u(\theta-\tau) e^{-2 \pi W(\theta-\tau)} d \theta \\
& =\pi W\left[e^{-2 \pi W|t-\tau|}-e^{2 \pi W\left(t+\tau-2 T_{o}\right)}\right]
\end{aligned}
$$


is certainly not time-invariant. However, we may specialize general formulas or apply Franks' method [4]. In both cases, taking the second derivative of the equation

$$
\lambda \psi(t)=\int_{0}^{T} \psi(\tau) \pi W\left[e^{-2 \pi W|t-\tau|}-e^{2 \pi W\left(t+\tau-2 T_{o}\right)}\right] d \tau
$$

we obtain the differential equation

$$
\lambda \frac{d^{2} \psi(t)}{d t^{2}}=-(2 \pi W)^{2} \psi(t)+\lambda(2 \pi W)^{2} \psi(t)
$$

The characteristic equation of this linear differential equation is

$$
1-\frac{s^{2}}{(2 \pi W)^{2}}-\frac{1}{\lambda}=0 .
$$

The roots are purely imaginary

$$
\sigma= \pm 2 \pi j W z, \quad \text { with } z=\sqrt{\frac{1}{\lambda}-1}
$$

and the complex form of the eigenfunctions is

$$
\psi(t)=C_{1} e^{-2 \pi j W z t}+D_{1} e^{2 \pi j W z t}
$$

while the real form is

$$
\psi(t)=C \cos (2 \pi W z t)+D \sin (2 \pi W z t) .
$$

Substituting one of these expressions into (13), we obtain for $z$ the following transcendental equation:

$$
\begin{aligned}
{\left[e^{-4 \pi W\left(T_{o}-T\right)}\left(1+z_{n}^{2}\right)+\left(1-z_{n}^{2}\right)\right] } & \sin \left(2 \pi W T z_{n}\right) \\
& +2 z_{n} \cos \left(2 \pi W T z_{n}\right)=0 .
\end{aligned}
$$

Let us rewrite this transcendental equation using the parametric representation for sine and cosine

$$
\begin{aligned}
\sin \left(2 \pi W T z_{n}\right) & =\frac{2 \tan \left(\pi W T z_{n}\right)}{1+\tan ^{2}\left(\pi W T z_{n}\right)} \\
\cos \left(2 \pi W T z_{n}\right) & =\frac{1-\tan ^{2}\left(\pi W T z_{n}\right)}{1+\tan ^{2}\left(\pi W T z_{n}\right)}
\end{aligned}
$$

to get the equation

$$
\begin{aligned}
& \tan ^{2}\left(\pi W T z_{n}\right) \\
& \quad-\left(\frac{1-z_{n}^{2}+e^{-4 \pi W\left(T_{o}-T\right)}\left(1+z_{n}^{2}\right)}{z_{n}}\right) \tan \left(\pi W T z_{n}\right)-1=0
\end{aligned}
$$

which splits into two "simpler" equations

$$
\begin{aligned}
\tan \left(\pi W T z_{n}\right)= & \frac{1-z_{n}^{2}+e^{-4 \pi W\left(T_{o}-T\right)}\left(1+z_{n}^{2}\right)}{2 z_{n}} \\
& \pm \sqrt{\left(\frac{1-z_{n}^{2}+e^{-4 \pi W\left(T_{o}-T\right)}\left(1+z_{n}^{2}\right)}{2 z_{n}}\right)^{2}+1}
\end{aligned}
$$

Note that when $T_{o} \rightarrow \infty$, we obtain Youla's solution [17]

$$
\left\{\begin{array}{rl}
z_{2 n-1} & =\cot \left(\pi W T z_{2 n-1}\right), \\
z_{2 n} & =-\tan \left(\pi W T z_{2 n}\right),
\end{array} \quad n=1,2, \cdots .\right.
$$

When $T_{o}=T$, it is better to solve the original equation, obtaining

$$
\tan \left(2 \pi W T z_{n}\right)=-z_{n}
$$

The real eigenfunctions are

$$
\psi_{n}(t)=A\left[z_{n} \cos \left(2 \pi W T z_{n}\right)+\sin \left(2 \pi W T z_{n}\right)\right]
$$

with the normalizing factor

$$
\begin{aligned}
A= & {\left[\frac{T}{2 \lambda_{n}}+\frac{1}{4 \pi W}-\frac{\cos \left(4 \pi W T z_{n}\right)}{4 \pi W}\right.} \\
& \left.-\frac{\left(1-z_{n}^{2}\right) \sin \left(4 \pi W T z_{n}\right)}{8 \pi W z_{n}}\right]^{-1 / 2} .
\end{aligned}
$$

Once $z_{n}$ is known, the corresponding eigenvalue $\lambda_{n}$ is obtained as

$$
\lambda_{n}=\frac{1}{1+z_{n}^{2}} .
$$

Results for $2 W T=1,2,4$ are reported in Table I, where we considered rectangular and sinusoidal pulses (see Section IV) as well as optimal signals. All input signals have unit energy. Both the energy transfer ratio and the energy ISI ratio have been evaluated for different combinations of the parameters. The results show the performance enhancement of optimal versus standard waveforms.

We also observe that two joint positive effects occur optimizing over $J=I$. First, the energy passing through the filter included in the time interval $I$ is increased, and secondly, the amount of interfering energy is decreased.

\section{A. The $t_{0}+I$ Case}

In this section we give a solution of Problem 1 for channels with a rational transfer function, taking the offset $t_{0}$ into account. Let us consider $J=\left[t_{0}, t_{0}+T\right]$. Consequently, $\mathcal{K}_{J}(t, \tau)$ is not time-invariant and it can be written as a difference

$$
\mathcal{K}_{J}(t, \tau)=\mathcal{K}_{0}(|t-\tau|)-\mathcal{K}_{\text {res }}(t, \tau)
$$

where $\mathcal{K}_{0}(t, \tau)$ is obtained as follows:

$$
\begin{aligned}
K_{0}(|t-\tau|) & =\int_{t_{0}}^{\infty} h(\theta-t) h(\theta-\tau) d \theta \\
& =\int_{\max \left\{t_{0}, t, \tau\right\}}^{\infty} \sum_{i, j} A_{i} A_{j} e^{\left(\alpha_{i}+\alpha_{j}\right) \theta-\alpha_{i} t-\alpha_{j} \tau} d \theta \\
& =\sum_{i, j}-\frac{A_{i} A_{j}}{\alpha_{i}+\alpha_{j}} e^{\left(\alpha_{i}+\alpha_{j}\right)\left(\max \left\{t_{0}, t, \tau\right\}\right)-\alpha_{i} t-\alpha_{j} \tau}
\end{aligned}
$$

and

$$
\mathcal{K}_{\text {res }}(t, \tau)=\int_{t_{0}+T}^{\infty} h(\theta-t) h(\theta-\tau) d \theta, \quad t, \tau \in[0, T]
$$

The kernel $\mathcal{K}_{0}(|t-\tau|)$ assumes two forms, depending on whether $t_{0}<\max \{t, \tau\}$ or $t_{0}>\max \{t, \tau\}$, which are either

$$
\begin{array}{r}
\mathcal{K}_{0}(|t-\tau|)=-\sum_{i, j} \frac{A_{i} A_{j}}{\alpha_{i}+\alpha_{j}} e^{\frac{\alpha_{i}+\alpha_{j}}{2}|t-\tau|-\frac{\alpha_{i}-\alpha_{j}}{2}(t-\tau)}, \\
t_{0}<\max \{t, \tau\}
\end{array}
$$


TABLE I

Energy Transfer for a Single-Pole Low-Pass Filter with "Optimal," "Rectangular," and "Sinusoidal" Signals. Note That $\lambda=\mathcal{E}_{J}$ Since We Assume $\mathcal{E}_{I}=1$

\begin{tabular}{|c|c|c|c|c|c|c|c|}
\hline Waveforms & $i$ & $z_{i}$ & $\lambda_{i}$ & $t_{0 i}$ & $\mathcal{E}_{R}$ & $\mathcal{E}_{J}$ & $\eta(\%)$ \\
\hline \multicolumn{8}{|l|}{$2 W T=1$} \\
\hline Rectang. & 1 & & & \multirow{5}{*}{0.121} & 0.695 & 0.549 & 26.5 \\
\hline Sine & 1 & & & & 0.666 & 0.579 & 14.9 \\
\hline Opt. on $I$ & 1 & 0.787 & 0.6172 & & 0.680 & 0.6172 & 10.2 \\
\hline Opt. on $t_{0}+I$ & 1 & 0.720 & 0.6683 & & 0.7027 & 0.6586 & 6.7 \\
\hline Opt. on $R$ & 1 & 0.638 & 0.7105 & & 0.7105 & 0.5824 & 22.0 \\
\hline \multicolumn{8}{|l|}{$2 W T=2$} \\
\hline \multirow[t]{2}{*}{ Rectang. } & 1 & & & & 0.8410 & 0.7620 & 10.4 \\
\hline & 2 & & & & 0.5497 & 0.4830 & 13.8 \\
\hline $\operatorname{Sin}(\pi t)$ & 1 & & & & 0.8510 & 0.8254 & 3.1 \\
\hline $\operatorname{Sin}(2 \pi t)$ & 2 & & & & 0.5794 & 0.5398 & 7.3 \\
\hline \multirow[t]{2}{*}{ Opt. on $I$} & 1 & 0.4347 & 0.8410 & & 0.8598 & 0.8410 & 2.2 \\
\hline & 2 & 0.8847 & 0.5609 & & 0.5969 & 0.5609 & 6.4 \\
\hline \multirow[t]{2}{*}{ Opt. on $t_{0}+I$} & 1 & 0.408 & 0.8591 & 0.133 & 0.8650 & 0.8516 & 1.6 \\
\hline & 2 & 0.8324 & 0.5860 & 0.117 & 0.6139 & 0.5860 & 4.8 \\
\hline \multirow[t]{2}{*}{ Opt. on $R$} & 1 & 0.3834 & 0.8718 & & 0.8718 & 0.8233 & 5.9 \\
\hline & 2 & 0.7875 & 0.6172 & & 0.6172 & 0.5395 & 14.4 \\
\hline \multicolumn{8}{|l|}{$2 W T=4$} \\
\hline \multirow[t]{4}{*}{ Rectang. } & 1 & & & & 0.9204 & 0.8806 & 4.5 \\
\hline & 2 & & & & 0.7619 & 0.7224 & 5.5 \\
\hline & 3 & & & & 0.6164 & 0.5832 & 5.7 \\
\hline & 4 & & & & 0.4831 & 0.4496 & 7.4 \\
\hline $\operatorname{Sin}(\pi \mathfrak{t})$ & 1 & & & & 0.9500 & 0.9456 & 0.46 \\
\hline $\operatorname{Sin}(2 \pi t)$ & 2 & & & & 0.8255 & 0.8127 & 1.57 \\
\hline $\operatorname{Sin}(3 \pi t)$ & 3 & & & & 0.6767 & 0.6583 & 2.8 \\
\hline $\operatorname{Sin}(4 \pi t)$ & 4 & & & & 0.5398 & 0.5199 & 3.8 \\
\hline \multirow[t]{4}{*}{ Opt. on $I$} & 1 & 0.2318 & 0.9490 & & 0.9526 & 0.9490 & 0.4 \\
\hline & 2 & 0.4653 & 0.8220 & & 0.8329 & 0.8220 & 1.3 \\
\hline & 3 & 0.7013 & 0.6703 & & 0.6870 & 0.6703 & 2.5 \\
\hline & 4 & 0.9400 & 0.5309 & & 0.5499 & 0.5309 & 3.6 \\
\hline \multirow[t]{4}{*}{ Opt. on $t_{0}+I$} & 1 & 0.2243 & 0.9519 & 0.067 & 0.9553 & 0.9519 & 0.36 \\
\hline & 2 & 0.4506 & 0.8312 & 0.067 & 0.8405 & 0.8312 & 1.1 \\
\hline & 3 & 0.6822 & 0.6824 & 0.067 & 0.6971 & 0.6824 & 2.2 \\
\hline & 4 & 0.9197 & 0.5417 & 0.067 & 0.5600 & 0.5417 & 3.4 \\
\hline \multirow[t]{4}{*}{ Opt. on $R$} & 1 & 0.2161 & 0.9553 & & 0.9553 & 0.9441 & 1.2 \\
\hline & 2 & 0.4347 & 0.8410 & & 0.8410 & 0.8094 & 3.9 \\
\hline & 3 & 0.6574 & 0.6982 & & 0.6982 & 0.6560 & 6.4 \\
\hline & 4 & 0.8847 & 0.5609 & & 0.5909 & 0.5205 & 7.7 \\
\hline
\end{tabular}

or

$$
\begin{array}{r}
\mathcal{K}_{0}(|t-\tau|)=\sum_{i, j} \frac{A_{i} A_{j}}{\alpha_{i}+\alpha_{j}} e^{\left(\alpha_{i}+\alpha_{j}\right) t_{0}-\alpha_{i} t-\alpha_{j} \tau}, \\
t_{0}>\max \{t, \tau\} .
\end{array}
$$

It follows that if $t, \tau \in\left[t_{0}, T\right]$ then eigenfunctions over this interval must have the form (11) and this is maintained over the whole interval $[0, T]$. The conclusion is that the eigenfunctions have the same form as for $t_{0}=0$ and the eigenvalues are obtained from the same equation with $t_{0}$ as a parameter. Finally, optimal $t_{0}$ and $\lambda$ 's are obtained from the system composed of (12) and the equation

$$
y\left(t_{0}\right)^{2}=y\left(t_{0}+T\right)^{2}
$$

as required by Proposition 2.2.

Example 1 (cont.): For instance, in the case of a first-order filter, the received signal $y(t)$ evaluated at times $t_{0}$ and $t_{0}+T$ gives

$$
\left\{\begin{aligned}
y\left(t_{0}\right) & =A \sin 2 \pi z_{n} W t_{0} \\
y\left(t_{0}+T\right) & =A e^{-2 \pi W t_{0}} \sin 2 \pi z_{n} W T .
\end{aligned}\right.
$$

Hence we obtain the equations

$$
\begin{aligned}
{\left[\left(1-z_{n}^{2}\right)+e^{-4 \pi W t_{0 n}}\left(1+z_{n}^{2}\right)\right] \tan 2 \pi z_{n} W T+2 z_{n} } & =0 \\
e^{-4 \pi W t_{0 n}} \sin ^{2} 2 \pi z_{n} W T-\sin ^{2} 2 \pi z_{n} W t_{0 n} & =0
\end{aligned}
$$

where we wrote $t_{0 n}$ for $t_{0}$ as the optimal delay which depends on the order $n$ of the eigenvalue $\lambda_{n}=1 /\left(1+z_{n}^{2}\right)$.

These two equations suggest an iterative method for calculating $t_{0 n}$ and $z_{n}$. Starting with a tentative value of $z_{n}^{(0)}$, near to the value obtained for $t_{0 n}=0$, from the first equation we estimate $t_{0 n}^{(1)}$ as

$$
t_{0 n}^{(1)}=\frac{-1}{4 \pi W} \ln \frac{-2 z_{n}^{(0)} \cot 2 \pi z_{n} W T-\left(1-\left[z_{n}^{(0)}\right]^{2}\right)}{1+\left[z_{n}^{(0)}\right]^{2}} .
$$

Then we substitute into the second equation and solve for $z_{n}^{(1)}$. We compute $t_{0 n}^{(2)}$ using the first equation, and so on.

\section{Numerical RESUlts}

Numerical computation of eigenvalues and eigenfunctions is generally a critical issue because of possible numerical instability. This motivates resorting to analytic solutions in special cases to check the accuracy of the numerical results obtained. The computation of the eigenvalues is amenable to the solution of a transcendental equation.

We will consider the calculation of eigenvalues and eigenfunctions for channels with rational transfer functions, defining the Shannon dimension of a band-limited and time-limited transmission system as $2 W T=c$ [13]. We explicitly consider three cases $c=1,2,4$, which represent the situations frequently occurring in present-day and will occur in future communication systems, and we compute the energy transfer ratio and the energy ISI ratio for each signal. Both figures are also evaluated for standard base signal sets which are currently used in digital communications. In particular, we will refer to "rectangular" and "sinusoidal" sets, namely,

$$
\left\{\begin{aligned}
x_{1}(t) & =u(t)-u(t-T) \\
x_{2}(t)=u(t)-2 u(t-T / 2)+u(t-T) & \\
x_{3}(t)=u(t)-2 u(t-T / 4)+ & 2 u(t-3 T / 4)-u(t-T) \\
x_{4}(t)=u(t)-2 u(t-T / 4)+ & 2 u(t-T / 2) \\
- & 2 u(t-3 T / 4)+u(t-T) \\
\text { ("rectangular" set) } & (15)
\end{aligned}\right.
$$

and

$$
\left\{\begin{array}{l}
x_{1}(t)=\sqrt{2} \sin (\pi t / T)[u(t)-u(t-T)] \\
x_{2}(t)=\sqrt{2} \sin (2 \pi t / T)[u(t)-u(t-T)] \\
x_{3}(t)=\sqrt{2} \sin (3 \pi t / T)[u(t)-u(t-T)] \\
x_{4}(t)=\sqrt{2} \sin (4 \pi t / T)[u(t)-u(t-T)] \\
\text { ("sinusoidal" set). }
\end{array}\right.
$$

Tables I-III report numerical results obtained by the analytic methods proposed.

Table I contains the energy transfer ratios $\mathcal{E}_{R}, \mathcal{E}_{J}$, and the energy ISI ratio $\eta$ for the single-pole linear filter with the 
TABLE II

ENergy Transfer for the IdEAL Low-PASS Filter with the“Optimal," "Rectangular," and "Sinusoidal” Signal Sets

\begin{tabular}{|c|c|c|c|c|c|c|}
\hline & \multicolumn{6}{|c|}{ Optimal signal set } \\
\hline & \multicolumn{3}{|c|}{$J=R$} & \multicolumn{3}{|c|}{$J=I$} \\
\hline$i$ & $c=1$ & $c=2$ & $c=4$ & $c=1$ & $c=2$ & $c=4$ \\
\hline 1 & 0.7833 & 0.9810 & 0.9999 & 0.6135 & 0.9623 & 0.9998 \\
\hline 2 & & 0.7515 & 0.9878 & & 0.5630 & 0.9757 \\
\hline 3 & & & 0.9530 & & & 0.9083 \\
\hline 4 & & & 0.7379 & & & 0.5323 \\
\hline & \multicolumn{6}{|c|}{ Rectangular signal set } \\
\hline 1 & 0.7737 & 0.9028 & 0.9499 & 0.5908 & 0.8315 & 0.8556 \\
\hline 2 & & 0.6446 & 0.8557 & & 0.4578 & 0.7735 \\
\hline 3 & & & 0.7340 & & & 0.6328 \\
\hline 4 & & & 0.5531 & & & 0.3580 \\
\hline & \multicolumn{6}{|c|}{ Sinusoidal signal set } \\
\hline 1 & 0.6984 & 0.9701 & 0.9980 & 0.5323 & 0.9111 & 0.9184 \\
\hline 2 & & 0.6327 & 0.9892 & & 0.4463 & 0.9074 \\
\hline 3 & & & 0.9404 & & & 0.8251 \\
\hline 4 & & & 0.5836 & & & 0.3732 \\
\hline
\end{tabular}

optimal, "rectangular" and "sinusoidal" signal sets. $J$ is chosen as $t_{0}+I$ in order to maximize $\mathcal{E}_{J}$ with respect to $t_{0}$. The results show that the performance of the "sinusoidal" signal set is very close to that of the optimal set for $c=2$ and 4 in terms of $\mathcal{E}_{R}$ and $\mathcal{E}_{J}$. A larger difference is observed for the "rectangular" signal set. Focusing on the energy ISI ratio, we note a larger advantage in using the optimal signal set.

Tables II and III report the energy transfer ratio for the ideal low-pass filter and the second-order Butterworth filter, respectively, with the optimal, "rectangular" and "sinusoidal" signal sets. The energy transfer ratios for the optimal signal sets have been calculated by using the analytic results obtained in Section III and the appendices.

\section{A. Numerical Integration}

When the integral equation (5) is not analytically solvable or algebraic manipulations are too hard, we revert to direct numerical methods. One simple technique consists in substituting the integral in (5) by its rectangular approximation. We then get the following matrix eigenvalue problem:

$$
\sum_{i=1}^{N} \mathcal{K}_{J}\left(t_{j}, t_{i}\right) x\left(t_{i}\right) \Delta t_{i}=\lambda x\left(t_{j}\right), \quad j=1,2, \cdots, N .
$$

The convergence of the sampled values $x\left(t_{i}\right)$ depends on the number and location of the nodes $t_{i}$ 's which can be selected in two ways: either matched to the kernel $\mathcal{K}_{J}(t, \tau)$ or simply uniformly on the interval $I$. For further reference on this subject see [10].

\section{COMMENTS AND REMARKS}

In this paper we have considered the problem of computing sets of signals which convey the maximum amount of energy included in the signaling time interval. Reference has been
TABLE III

Energy Transfer Ratio for a Second-Order Butterworth Filter with "Optimal," "Rectangular," and "Sinusoidal” Signal Sets

\begin{tabular}{|c|c|c|c|c|c|c|}
\hline & \multicolumn{6}{|c|}{ Optimal signal set } \\
\hline & \multicolumn{3}{|c|}{$J=R$} & \multicolumn{3}{|c|}{$J=I$} \\
\hline$i$ & $c=1$ & $c=2$ & $c=4$ & $c=1$ & $c=2$ & $c=4$ \\
\hline 1 & 0.75470 & 0.93740 & 0.99130 & 0.49238 & 0.88189 & 0.98727 \\
\hline 2 & & 0.67670 & 0.93960 & & 0.50027 & 0.91222 \\
\hline 3 & & & 0.80590 & & & 0.73270 \\
\hline 4 & & & 0.60510 & & & 0.50016 \\
\hline & \multicolumn{6}{|c|}{ Rectangular signal set } \\
\hline 1 & 0.74071 & 0.88838 & 0.94372 & 0.37714 & 0.71722 & 0.85929 \\
\hline 2 & & 0.59304 & 0.83305 & & 0.39960 & 0.74630 \\
\hline 3 & & & 0.68612 & & & 0.58773 \\
\hline 4 & & & 0.49996 & & & 0.40489 \\
\hline & \multicolumn{6}{|c|}{ Sinusoidal signal set } \\
\hline 1 & 0.69087 & 0.92025 & 0.99003 & 0.37716 & 0.83177 & 0.97911 \\
\hline 2 & & 0.61162 & 0.93097 & & 0.44046 & 0.88587 \\
\hline 3 & & & 0.77576 & & & 0.69465 \\
\hline 4 & & & 0.55628 & & & 0.47185 \\
\hline
\end{tabular}

made to the class of linear filters with rational transfer functions. We have shown that the computation of the eigenvalues can be traced back to the solution of a transcendental equation. We have also shown that the eigenfunctions are linear combinations of possibly complex exponential time functions with exponent coefficients which are algebraic functions of the eigenvalues. We have also computed eigenvalues and eigenfunctions by direct numerical integration and numerical optimization. The agreement between the results obtained with the two methods validates the "numerical" technique, which is preferable or unavoidable when dealing with complex rational transfer functions.

Knowing the form of the eigenfunctions is also important for practical implementation. It may be easier to generate linear combinations of exponential and trigonometric functions rather than to interpolate inaccurate samples obtained by numerical integrations.

Finally, we introduced the energy ISI ratio, a second figure of merit besides the classical energy transfer ratio, which is significant if the optimization aims at reducing ISI. In conclusion we will discuss an important feature of optimal signals which may affect the design of multidimensional signal sets.

\section{A. Application to Multidimensional Modulations}

A possible application of the signals which solve Problem 1 is the definition of a base signal set $\left\{\psi_{i}(t)\right\}_{i=1}^{n}$ with $t \in[0, T]$ in multidimensional modulations. The set of $M$ signals $\left\{s_{j}(t)\right\}_{j=1}^{M}$ is generated from the set of $n$-dimensional $\left\{\boldsymbol{X}_{j}\right\}_{j=1}^{M}$ as

$$
s_{j}(t)=\sum_{i=1}^{n} x_{i j} \psi_{i}(t), \quad j=1, \cdots, M
$$


where $x_{i j}$ is the $i$ th component of $\boldsymbol{X}_{j}$. The orthogonal base signals $\psi_{i}(t)$ are attenuated and rotated in different ways through the channel. Hence, the received signals, which are corrupted by noise and ISI, are also asymmetrical. To get rid of these distortions, we can use $n$ orthogonal eigenfunctions - obtained as the solution of Problem 1 with $J=I$ - as base signals conveniently rescaled so as to have energies $1 / \lambda_{i}$. At the channel output, the received signals

$$
\phi_{i}(t)=\int_{J} h(t-\tau) \psi_{i}(\tau) d \tau
$$

are orthonormal. In fact we have

$$
\begin{aligned}
& \int_{J} \phi_{i}(t) \phi_{j}(t) d t \\
& =\int_{J} \int_{I} \psi_{j}(u) h(t-u) d u \int_{I} \psi_{i}(v) h(t-v) d v d t \\
& =\int_{I} \int_{I} \psi_{j}(u) \psi_{i}(v) \int_{J} h(t-u) h(t-v) d t d v d u \\
& =\int_{I} \int_{I} \mathcal{K}_{J}(u, v) \psi_{j}(u) \psi_{i}(v) d v d u \\
& =\lambda_{j} \int_{I} \psi_{j}(v) \psi_{i}(v) d v=\delta_{i j} .
\end{aligned}
$$

Therefore, the set of signals that convey the maximum energy over the time interval $J$ gives also the minimum ISI, preserves orthogonality, and, on suitable rescaling, maintains the symmetry of the $n$-dimensional point constellations. Thus further use of equalization will make ISI negligible.

\section{B. Conclusion}

The results provided in this paper show that optimization is convenient for several reasons.

1) With respect to standard solutions, observed gains are not marginal.

2) Optimal signals preserve orthogonality after filtering.

3) For large $2 W T$ sinusoidal pulses are nearly optimal from the ISI and energy transfer points of view, but they do not maintain the original orthogonality after filtering.

4) Using optimal base signals we can design signal sets for multidimensional modulations preserving symmetry at the receiver input.

In conclusion, the search for signals with a maximum energy transfer over a finite interval certainly deserves further investigation. Future work should focus on nonlinearity effects, fading, and other impairments which affect cable and mobile channels.

\section{APPENDIX A}

\section{THE IDEAL LOW-PASS CHANNEL}

The ideal low-pass channel with bandwidth $W$ was considered as a "hard" frequency limiter [13] in the search for a rigorous proof of Shannon's heuristic that the dimension of a set of time- and frequency-limited signals is approximately $2 W T$ [12]. The input pulse is defined over $I=[0, T]$ and for infinite output time interval the kernel of the integral equation
(5) is

$$
\mathcal{K}_{J}(u, v)=\frac{\sin [2 \pi W(u-v)]}{\pi(u-v)} .
$$

Functions satisfying (5) were obtained by the observation that the integral operator

$$
\mathcal{I}: \phi(t) \rightarrow \int_{0}^{T} \mathcal{K}_{J}(t, \tau) \phi(\tau) d \tau
$$

and the differential operator

$$
\mathcal{D}: \phi(t) \rightarrow\left[t(T-t) \phi^{\prime}(t)\right]^{\prime}-4 \pi^{2} W^{2} t(T-t) \phi(t)
$$

commute. Hence, both operators have the same eigenfunctions which are a scaled and translated version of prolate spheroidal wave functions. An extensive account of the analytic and asymptotic properties of these functions and related eigenvalues is given in [6], [7], [13], and [14].

When the output time interval has finite duration $T$, the kernel can be expressed by using sine and cosine integral functions

$$
\begin{aligned}
\mathcal{K}_{J}(u, v)= & \frac{\cos (2 \pi W(u-v))}{2 \pi^{2}(u-v)}[\operatorname{Cin}(4 \pi W u)-\operatorname{Cin}(4 \pi W v) \\
& -\operatorname{Cin}(4 \pi W(T-u))+\operatorname{Cin}(4 \pi W(T-v))] \\
& +\frac{\sin (2 \pi W(u-v))}{2 \pi^{2}(u-v)}[\operatorname{Si}(4 \pi W u)+\operatorname{Si}(4 \pi W v) \\
& +\operatorname{Si}(4 \pi W(T-u))+\operatorname{Si}(4 \pi W(T-v))]
\end{aligned}
$$

where

$$
\begin{aligned}
\operatorname{Cin}(x)=\int_{0}^{x} \frac{1-\cos t}{t} d t=\gamma+\ln x-\operatorname{Ci}(x) \\
\quad \text { and } \operatorname{Si}(x)=\int_{0}^{x} \frac{\sin t}{t} d t .
\end{aligned}
$$

$\gamma=0.577$ is the Euler constant. Comparative numerical results are given in Table II.

\section{APPENDIX B YOULA'S PROCEDURE [17]}

1) Set $K(s)=H(s) H(-s)=N\left(s^{2}\right) / D\left(s^{2}\right)$. Let $2 m$ and $2 n$ be the degrees of $N\left(s^{2}\right)$ and $D\left(s^{2}\right)$, respectively, with $m<n$.

2) Let $\pm \mu_{i}, i=1, \cdots, n$ be the roots of $D\left(s^{2}\right)=0$ ordered according to the increasing positive real part $0 \leq \operatorname{Re} \mu_{1} \leq \operatorname{Re} \mu_{2} \ldots \leq \operatorname{Re} \mu_{n}$ and set

$$
D^{+}(s)=\prod_{i=1}^{n}\left(s+\mu_{i}\right) \quad D^{-}(s)=\prod_{i=1}^{n}\left(s-\mu_{i}\right) .
$$

3) Let $\sigma_{i}(\lambda)$ be the roots of $N\left(s^{2}\right)-\lambda D\left(s^{2}\right)=0$ with positive real part and set

$$
x_{i}(\lambda)=\exp \left(-\sigma_{i}(\lambda) T\right) \frac{D^{-}\left(\sigma_{i}(\lambda)\right)}{D^{+}\left(\sigma_{i}(\lambda)\right)}, \quad i=1, \cdots, n .
$$

4) Find the roots $\lambda_{r}^{( \pm)}, r=1,2, \cdots$, of the following two transcendental equations:

$$
\operatorname{det}\left(\left[\left(1 \pm(-1)^{j} x_{i}(\lambda)\right)\left(\sigma_{i}(\lambda)\right)^{j-1}\right]_{i, j=1}^{n}\right)=0 .
$$


Since $\lambda_{r}^{( \pm)}$are positive real numbers, without loss of generality we can assume $\lambda_{r}^{( \pm)}>\lambda_{r+1}^{( \pm)}$.

5) The eigenfunctions are found by solving the linear equation system

$$
\begin{aligned}
\sum_{j=0}^{n-1}\left(1 \pm(-1)^{j} x_{i}\left(\lambda_{r}^{( \pm)}\right)\right)\left(\sigma_{i}\left(\lambda_{r}^{( \pm)}\right)\right)^{j} p_{j} & =0, \\
i & =1, \cdots, n
\end{aligned}
$$

in the unknown coefficients $p_{j}$ and can be written as

$$
x_{r}(t)=\mathcal{L}^{-1}\left\{\frac{D^{+}(s) \sum_{j=0}^{n-1} p_{j} s^{j}}{N\left(s^{2}\right)-\lambda_{r}^{( \pm)} D\left(s^{2}\right)}\right\}, \quad r=1,2, \cdots .
$$

Each $x_{r}(t)$ turns out to be a linear combination of exponentials in $|t|$.

\section{APPENDIX C \\ SECOND-ORDER BUTTERWORTH FILTER}

Butterworth filters are better characterized by $K(s)$ and the transfer function can be obtained, with the condition that the real part of the roots of $H(s)$ is negative, from

$$
K(s)=H(s) H(-s)=\frac{1}{1+s^{4} /(2 \pi W)^{4}}
$$

where $W$ is the $3-\mathrm{dB}$ bandwidth. Let us apply Youla's procedure by way of example to recall a solution for infinite output interval. Later we will apply our procedure to solve for finite output interval.

We have obviously

$$
N\left(s^{2}\right)=1 \quad \text { and } \quad D\left(s^{2}\right)=1+s^{4} /(2 \pi W)^{4}
$$

and

$$
\left\{\begin{array}{l}
D^{+}(s)=1+\sqrt{2} \frac{s}{2 \pi W}+\frac{s^{2}}{(2 \pi W)^{2}} \\
D^{-}(s)=1-\sqrt{2} \frac{s}{2 \pi W}+\frac{s^{2}}{(2 \pi W)^{2}} .
\end{array}\right.
$$

The roots $\sigma_{i}$ of the equation

$$
1-\lambda\left(1+\frac{s^{4}}{(2 \pi W)^{4}}\right)=0
$$

depend on the eigenvalues $\lambda$. Since $\lambda<1$ we have

$$
\left\{\begin{array}{l}
\sigma_{1}= \pm 2 j \pi W z, \\
\sigma_{2}= \pm 2 \pi W z,
\end{array} \text { where } z=\sqrt[4]{\frac{1}{\lambda}-1} .\right.
$$

Using $\sigma_{1}$ and $\sigma_{2}$ both with positive signs we may define

$$
\begin{aligned}
& x_{1}=e^{-j 2 \pi z W T} \frac{1-j \sqrt{2} z-z^{2}}{1+j \sqrt{2} z-z^{2}} \\
& x_{2}=e^{-2 \pi z W T} \frac{1-\sqrt{2} z+z^{2}}{1+\sqrt{2} z+z^{2}}
\end{aligned}
$$

by means of which we write out two determinants, one for each choice of sign (upper or lower):

$$
\Delta^{ \pm}=\left|\begin{array}{ll}
\left(1 \mp x_{1}\right) & \left(1 \pm x_{1}\right) 2 j \pi W z \\
\left(1 \mp x_{2}\right) & \left(1 \pm x_{2}\right) 2 \pi W z
\end{array}\right| .
$$

After some calculations, we get two transcendental equations for $z$

$$
\begin{aligned}
& e^{-2 \pi z W T}\left(\left(z^{2}-\sqrt{2} z-1\right)\left(z^{2}-\sqrt{2} z+1\right) \cos (\pi z W T)\right. \\
& \left.\quad+\left(z^{2}+\sqrt{2} z-1\right)\left(z^{2}-\sqrt{2} z+1\right) \sin (\pi z W T)\right) \\
& \quad-\left(z^{2}+\sqrt{2} z-1\right)\left(z^{2}+\sqrt{2} z+1\right) \cos (\pi z W T) \\
& \quad+\left(z^{2}+\sqrt{2} z+1\right)\left(z^{2}-\sqrt{2} z-1\right) \sin (\pi z W T)=0 \\
& e^{-2 \pi z W T}\left(\left(z^{2}+\sqrt{2} z-1\right)\left(z^{2}-\sqrt{2} z+1\right) \cos (\pi z W T)\right. \\
& \left.\quad-\left(z^{2}-\sqrt{2} z-1\right)\left(z^{2}-\sqrt{2} z+1\right) \sin (\pi z W T)\right) \\
& \quad-\left(z^{2}+\sqrt{2} z+1\right)\left(z^{2}-\sqrt{2} z-1\right) \cos (\pi z W T) \\
& \quad-\left(z^{2}+\sqrt{2} z-1\right)\left(z^{2}+\sqrt{2} z+1\right) \sin (\pi z W T)=0 .
\end{aligned}
$$

Let $z_{n}$ denote the sequence of roots in increasing order $0<$ $z_{1}<\cdots<z_{n}<\cdots$, to which corresponds the eigenvalue $\lambda_{n}=1 /\left(1+z_{n}^{4}\right)$ and the eigenfunction

$$
\begin{aligned}
x_{n}(t)= & A_{1} e^{2 \pi W z_{n}|t|}+A_{2} e^{-2 \pi W z_{n}|t|} \\
& +A_{3} e^{2 j \pi W z_{n}|t|}+A_{4} e^{-2 j \pi W z_{n}|t|}
\end{aligned}
$$

Finally, let us consider the finite output interval $J=I$. Let $\alpha_{1}$ and $\alpha_{2}$ denote the roots of $D^{+}(s)$ and $\sigma_{1}$ and $\sigma_{2}$ as defined in (17). From (12) we get a homogeneous linear system with coefficients determinat $\Delta=\left|B_{i j}\right|$ where

$$
\begin{aligned}
B_{11}= & \frac{1}{\alpha_{1}-\sigma_{1}} \quad B_{12}=\frac{1}{\alpha_{1}-\sigma_{2}} \\
B_{13}= & \frac{1}{\alpha_{1}+\sigma_{1}} \quad B_{14}=\frac{1}{\alpha_{1}+\sigma_{2}} \\
B_{21}= & \frac{1}{\alpha_{2}-\sigma_{1}} \quad B_{22}=\frac{1}{\alpha_{2}-\sigma_{2}} \\
B_{23}= & \frac{1}{\alpha_{2}+\sigma_{1}} \quad B_{24}=\frac{1}{\alpha_{2}+\sigma_{2}} \\
B_{31}= & e^{2 \pi z W T}\left[\frac{1}{2 \alpha_{1}\left(\alpha_{1}+\sigma_{1}\right)}+\frac{1}{2 \alpha_{1}\left(\alpha_{1}-\sigma_{1}\right)}\right. \\
& \left.-\frac{1}{\left(\alpha_{1}+\alpha_{2}\right)\left(\alpha_{1}+\sigma_{1}\right)}-\frac{1}{\left(\alpha_{1}+\alpha_{2}\right)\left(\alpha_{2}-\sigma_{1}\right)}\right] \\
& -e^{\alpha_{1} T} \frac{1}{2 \alpha_{1}\left(\alpha_{1}-\sigma_{1}\right)}+e^{\alpha_{2} T} \frac{1}{\left(\alpha_{1}+\alpha_{2}\right)\left(\alpha_{1}-\sigma_{1}\right)} \\
B_{32}= & e^{2 j \pi z W T}\left[\frac{1}{2 \alpha_{1}\left(\alpha_{1}+\sigma_{1}\right)}+\frac{1}{2 \alpha_{1}\left(\alpha_{1}-\sigma_{1}\right)}\right. \\
& \left.-\frac{1}{\left(\alpha_{1}+\alpha_{2}\right)\left(\alpha_{1}+\sigma_{1}\right)}-\frac{1}{\left(\alpha_{1}+\alpha_{2}\right)\left(\alpha_{2}-\sigma_{1}\right)}\right] \\
& -e^{\alpha_{1} T} \frac{1}{2 \alpha_{1}\left(\alpha_{1}-\sigma_{1}\right)}+e^{\alpha_{2} T} \frac{1}{\left(\alpha_{1}+\alpha_{2}\right)\left(\alpha_{1}-\sigma_{1}\right)} \\
B_{33}= & e^{-2 \pi z W T}\left[\frac{1}{2 \alpha_{1}\left(\alpha_{1}+\sigma_{1}\right)}+\frac{1}{2 \alpha_{1}\left(\alpha_{1}-\sigma_{1}\right)}\right. \\
& \left.-\frac{1}{\left(\alpha_{1}+\alpha_{2}\right)\left(\alpha_{1}+\sigma_{1}\right)}-\frac{1}{\left(\alpha_{1}+\alpha_{2}\right)\left(\alpha_{2}-\sigma_{1}\right)}\right] \\
& -e^{\alpha_{1} T} \frac{1}{2 \alpha_{1}\left(\alpha_{1}-\sigma_{1}\right)}+e^{\alpha_{2} T} \frac{1}{\left(\alpha_{1}+\alpha_{2}\right)\left(\alpha_{1}-\sigma_{1}\right)}
\end{aligned}
$$




$$
\begin{aligned}
& B_{34}=e^{-2 j \pi z W T}\left[\frac{1}{2 \alpha_{1}\left(\alpha_{1}+\sigma_{1}\right)}+\frac{1}{2 \alpha_{1}\left(\alpha_{1}-\sigma_{1}\right)}\right. \\
& \left.-\frac{1}{\left(\alpha_{1}+\alpha_{2}\right)\left(\alpha_{1}+\sigma_{1}\right)}-\frac{1}{\left(\alpha_{1}+\alpha_{2}\right)\left(\alpha_{2}-\sigma_{1}\right)}\right] \\
& -e^{\alpha_{1} T} \frac{1}{2 \alpha_{1}\left(\alpha_{1}-\sigma_{1}\right)}+e^{\alpha_{2} T} \frac{1}{\left(\alpha_{1}+\alpha_{2}\right)\left(\alpha_{1}-\sigma_{1}\right)} \\
& B_{41}=e^{2 \pi z W T}\left[\frac{1}{2 \alpha_{1}\left(\alpha_{1}+\sigma_{1}\right)}+\frac{1}{2 \alpha_{1}\left(\alpha_{1}-\sigma_{1}\right)}\right. \\
& \left.-\frac{1}{\left(\alpha_{1}+\alpha_{2}\right)\left(\alpha_{1}+\sigma_{1}\right)}-\frac{1}{\left(\alpha_{1}+\alpha_{2}\right)\left(\alpha_{2}-\sigma_{1}\right)}\right] \\
& -e^{\alpha_{1} T} \frac{1}{2 \alpha_{1}\left(\alpha_{1}-\sigma_{1}\right)}+e^{\alpha_{2} T} \frac{1}{\left(\alpha_{1}+\alpha_{2}\right)\left(\alpha_{1}-\sigma_{1}\right)} \\
& B_{42}=e^{2 j \pi z W T}\left[\frac{1}{2 \alpha_{1}\left(\alpha_{1}+\sigma_{1}\right)}+\frac{1}{2 \alpha_{1}\left(\alpha_{1}-\sigma_{1}\right)}\right. \\
& \left.-\frac{1}{\left(\alpha_{1}+\alpha_{2}\right)\left(\alpha_{1}+\sigma_{1}\right)}-\frac{1}{\left(\alpha_{1}+\alpha_{2}\right)\left(\alpha_{2}-\sigma_{1}\right)}\right] \\
& -e^{\alpha_{1} T} \frac{1}{2 \alpha_{1}\left(\alpha_{1}-\sigma_{1}\right)}+e^{\alpha_{2} T} \frac{1}{\left(\alpha_{1}+\alpha_{2}\right)\left(\alpha_{1}-\sigma_{1}\right)} \\
& B_{43}=e^{-2 \pi z W T}\left[\frac{1}{2 \alpha_{1}\left(\alpha_{1}+\sigma_{1}\right)}+\frac{1}{2 \alpha_{1}\left(\alpha_{1}-\sigma_{1}\right)}\right. \\
& \left.-\frac{1}{\left(\alpha_{1}+\alpha_{2}\right)\left(\alpha_{1}+\sigma_{1}\right)}-\frac{1}{\left(\alpha_{1}+\alpha_{2}\right)\left(\alpha_{2}-\sigma_{1}\right)}\right] \\
& -e^{\alpha_{1} T} \frac{1}{2 \alpha_{1}\left(\alpha_{1}-\sigma_{1}\right)}+e^{\alpha_{2} T} \frac{1}{\left(\alpha_{1}+\alpha_{2}\right)\left(\alpha_{1}-\sigma_{1}\right)} \\
& B_{44}=e^{-2 j \pi z W T}\left[\frac{1}{2 \alpha_{1}\left(\alpha_{1}+\sigma_{1}\right)}+\frac{1}{2 \alpha_{1}\left(\alpha_{1}-\sigma_{1}\right)}\right. \\
& \left.-\frac{1}{\left(\alpha_{1}+\alpha_{2}\right)\left(\alpha_{1}+\sigma_{1}\right)}-\frac{1}{\left(\alpha_{1}+\alpha_{2}\right)\left(\alpha_{2}-\sigma_{1}\right)}\right] \\
& -e^{\alpha_{1} T} \frac{1}{2 \alpha_{1}\left(\alpha_{1}-\sigma_{1}\right)}+e^{\alpha_{2} T} \frac{1}{\left(\alpha_{1}+\alpha_{2}\right)\left(\alpha_{1}-\sigma_{1}\right)} \text {. }
\end{aligned}
$$

Expanding $\Delta$, from the condition $\Delta=0$ we get the transcendental equation

$$
\begin{aligned}
& e^{2 \pi z W T}\left[\left(z^{2}+\sqrt{2} z+1\right)\left(z^{2}-1\right) \cos 2 \pi z W T\right. \\
& \left.\quad+\sqrt{2} z\left(z^{2}+\sqrt{2} z+1\right) \sin 2 \pi z W T\right] \\
& \quad+e^{-2 \pi z W T}\left[\left(z^{2}-\sqrt{2} z-1\right)\left(z^{2}+1\right) \cos 2 \pi z W T\right. \\
& \left.\quad+\sqrt{2} z\left(z^{2}-\sqrt{2} z+1\right) \sin 2 \pi z W T\right]+2\left(z^{4}+1\right)=0
\end{aligned}
$$

whose sequence of roots yields the eigenvalues for the output finite interval. Finally, the eigenfunctions are of the form

$$
\begin{aligned}
x_{n}(t)= & C_{1} e^{2 j \pi W z_{n} t}+D_{1} e^{-2 j \pi W z_{n} t} \\
& +C_{2} e^{2 \pi W z_{n} t}+D_{2} e^{-2 \pi W z_{n} t} .
\end{aligned}
$$

The results of this appendix have been used to compute the entries of Table III.

\section{REFERENCES}

[1] D. Courant and D. Hilbert, Mathematical Methods in Physics. Englewood Cliffs, NJ: Prentice-Hall, 1955.

[2] M. Elia and M. T. Galizia Angeli, "Autofunzioni di operatori lineari integrali associati ai filtri di Butterworth," presented at VII GMEL, Coimbra, Sept. 1985.

[3] _ "Eigenfunction of integral linear operators associated to second order filters," in Proc. IASTED Int. Symp. MIC'87 (Grindelwald, Switzerland), pp. 396-399.

[4] L. E. Franks, Signal Theory. Englewood Cliffs, NJ: Prentice-Hall, 1969.

[5] M. L. Honig and K. Steiglitz, "Maximizing the output energy of a linear channel with a time- and amplitude-limited input," IEEE Trans. Inform. Theory, vol. 38, pp. 1041-1052, May 1992

[6] H. J. Landau and H. O. Pollack, "Prolate spheroidal wave functions, Fourier analysis and uncertainty II," Bell Syst. Tech. J., vol. 40, pp. 65-84, 1961

[7] _ "Prolate spheroidal wave functions, Fourier analysis and uncertainty III," Bell Syst. Tech. J., vol. 41, pp. 1295-1336, 1962.

[8] P. G. Petrovsky, Lectures on the Theory of Integral Equations. Moscow, USSR: MIR, 1971.

[9] B. van der Pol and H. Bremmer, Operational Calculus Based on the Two Sided Laplace Transform. Cambridge, U.K.: Cambridge Univ. Press, 1955.

[10] W. H. Press, S. A. Teukolsky, W. T. Vetterling, and B. P. Flannery, Numerical Recipes in $C, 2$ nd ed. Cambridge, U.K.: Cambridge Univ. Press, 1992

[11] W. Schmeidler, Linear Operators in Hilbert Space. New York: Academic, 1965

[12] C. E. Shannon, "A mathematical theory of communication," Bell Sys. Tech. J., vol. 27, pp. 379-423, 1948

[13] D. Slepian, "Some comments on Fourier analysis, uncertainty and modeling," SIAM Rev., vol. 25, no. 3, pp. 379-393, July 1982.

[14] D. Slepian and H. O. Pollack, "Prolate spheroidal wave functions, Fourier analysis and uncertainty I," Bell Syst. Tech. J., vol. 40, pp. 43-64, 1961.

[15] A. E. Taylor and D. C. Lay, Introduction to Functional Analysis. New York: Wiley, 1980.

[16] N. Tugbay and E. Panayirci, "Energy optimization of band-limited Nyquist signals in the time domain," IEEE Trans. Commun., vol. COM35, pp. 427-434, Apr. 1987.

[17] D. C. Youla, "The solution of a homogeneous Wiener-Hopf integral equation occurring in the expansion of second-order stationary random functions," IRE Trans. Inform. Theory, vol. IT-3, pp. 187-193, Sept. 1957 